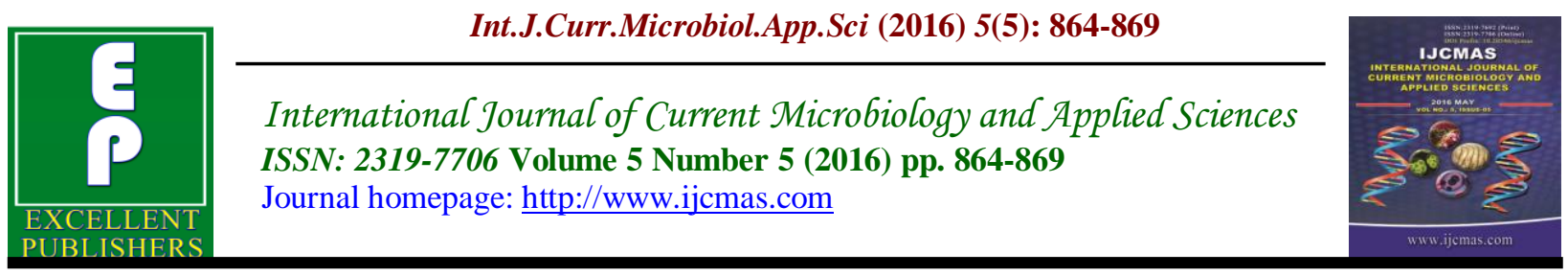

Original Research Article

http://dx.doi.org/10.20546/ijcmas.2016.505.089

\title{
Detection of Carbapenem Resistant Enterobacteriacae from Clinical Isolates
}

\author{
Akshaya Rao* and V.A. Indumathi \\ Department of Microbiology M.S. Ramaiah Medical College Bangalore, India \\ *Corresponding author
}

Keywords

Carbapenemases, KPC Chrom Agar, Modified Hodge

Test,

Blue Carba Test, Inhibitor Based Phenotypic Test.

Article Info

Accepted:

20 April 2016 Available Online: 10 May 2016
A B S T R A C T

Carbapenemases are $\beta$-lactamases that confer resistance to almost all $\beta$ Lactam antibiotics. There is an urgent need for identification of these enzymes. The main aim of this study includes to detect different Carbapenemase enzymes produced by Enterobacteriacae among clinical isolates using phenotypic tests. Isolates from various clinical samples that showed resistance to Imipenem and/or Meropenem by Kirby Bauer disc diffusion method were analysed over a period of 6 months. These isolates were screened using Modified Hodge test (MHT), KPC CHROM agar, and supplemental tests such as Blue Carba Test (BCT) and Inhibitor Based Phenotypic Test (IBPT) were done to detect different Carbapenemase enzymes. 60 out of $430(13.95 \%)$ strains were Carbapenem resistant Enterobacteriacae. Among the 4 tests performed MHT detected Carbapenemases in $36.6 \%$ and KPC CHROM agar in $96.6 \%$ isolates. Blue Carba Test showed $13.3 \%$ isolates as KPC/MBL phenotype and $3.3 \%$ as OXA phenotype. Inhibitor Based Phenotypic Test detected $8.3 \%$ isolates as KPC producers and 5\% as MBL producers. Among these four phenotypic tests KPC CHROM agar could be useful as a screening test for Carbapenemase detection, BCT and IBPT would be useful to differentiate these Carbapenemases.

\section{Introduction}

Carbapenem resistant Enterobacteriacae, or CRE are defined as those strains of organisms belonging to Enterobacteriacae family that are nonsusceptible to one of the following carbapenems: Meropenem, Imipenem or Doripenem and resistant to all of the following third-generation cephalosporins: Ceftriaxone, Cefotaxime and Ceftazidime.
Infections caused by these organisms are associated with high rates of morbidity and mortality (50\% according to some studies) (CDC, 2014). Delayed recognition and inappropriate treatment of severe infections caused by these organisms are associated with increased mortality. Patients can harbour CRE in their gastro intestinal tract and act as reservoirs for cross transmission. 
The drug resistance genes are often carried on mobile genetic elements and hence can easily transmit from person to person often via the hands of healthcare personnel or via contaminated medical equipment. (CDC, 2012) These genes confer high levels of resistance to Carbapenems and many other antimicrobials (fluoroquinolones and aminoglycosides), often leaving very limited therapeutic options.

Various mechanisms are responsible for the resistance the most common being the production of Carbapenemases a class of enzymes capable of hydrolysing carbapenems and other $\beta$-lactams. (Pravin, 2013) These enzymes have been detected in many different genera of Enterobacteriaceae, but most commonly in Escherichia coli and Klebsiella species. (CDC, 2012) The carbapenemases fall into three classes according to their amino acid sequence: (1) Ambler Class A serine carbapenemases (Ex: KPC, SME, IMI, NMC, GES, inhibited by clavulanic acid), (2) Class B metallo-beta-lactamases (Ex: IMP, VIM, SPM, GIM, and SIM, inhibited by metal chelators) and (3) Class D oxacillinase-type carbapenemases (OXA types) (Queenam et al., 2007). Other mechanisms of resistance includes the poor binding of Carbapenems to penicillinbinding proteins present in the bacteria, the over-expression of multidrug efflux pumps by the bacteria or lack of porins present in the bacterial cell membrane.

The risk of onward spread may vary with underlying resistance mechanisms or combinations of those. Hence, both EUCAST and CLSI indicate the value of screening tests and supplemental tests to detect and differentiate Carbapenemases for infection prevention and control purposes, and for local epidemiological investigations. (Public Health England, 2013)

\section{Materials and Methods}

This observational study was carried out in the Department of Microbiology, at a tertiary care teaching hospital for a period of 6 months from January 2015 to June 2015. The study was conducted after obtaining the ethical clearance from the institution ethical committee and informed consent from the patients.

Non repetitive isolates of Enterobacteriacae obtained from the clinical samples such as Blood, Sputum, Urine, Wound swabs, Catheter tips, Pus and other body fluids which showed resistance to Imipenem and/or Meropenem by Kirby Bauer disc diffusion method were collected.

These isolates were screened using KPC ChromAgar for the presence of Carbapenemase enzymes followed by supplemental tests such as Modified Hodge Test (Jacob Moran Gilada, 2011), Blue Carba Test (Modified Hodge Test for Carbapenemase Detection in Enterobacteriacae) and Inhibitor Based Phenotypic Test.

\section{Phenotypic Tests}

KPC CHROMagar -All the isolates were inoculated onto a chromogenic medium, HiCrome KPC Agar (Hi Media, Mumbai, India) supplemented with agents that inhibits the growth of Carbapenem-sensitive bacteria. Following 24 hours of incubation, Carbapenem-resistant Klebsiella and Escherichia coli appeared as cream coloured and red colonies respectively which is based on their specific enzymatic properties.

Modified Hodge Test, one among CLSI 2016 recomended tests was performed as per CDC guidelines (Modified Hodge Test for Carbapenemase Detection in 
Enterobacteriacae). A positive test showed a clover leaflike indentation of the E.coli ATCC 25922 growing along the test organism growth streak within the disk diffusion zone.

Other two supplemental tests such as Blue Carba Test (BCT) and Inhibitor Based Phenotypic Test (IBPT) were performed to detect and differentiate these Carbapenemases into different classes.

Blue Carba Test (BCT) is a modified version of Carba NP was performed as proposed by Pires et al., (2013). The test is based on the principle of direct detection of Carbapenem hydrolysis by Carbapenemaseproducing bacteria. It detects different classes of Carbapenemases that is KPC \& MBL in first 30mins and OXA types in 2 hours.

Inhibitor Based Phenotypic Test (IBPT) is based on the principle of inhibition of Carbapenemases by specific inhibitors. It was performed as described by Giske et al. (2010) In-house prepared discs containing Meropenem $(10 \mu \mathrm{g})$ alone, Meropenem (10 $\mu \mathrm{g})+\operatorname{EDTA}(730 \mu \mathrm{g})$, Meropenem $(10 \mu \mathrm{g})+$ Phenyl Boronic Acid $(600 \mu \mathrm{g})$ and Meropenem $(10 \mu \mathrm{g}+$ Cloxacillin $750 \mu \mathrm{g})$ were used on all CRE isolates for the detection of MBL, KPC and AmpC producers respectively. An increase of $5 \mathrm{~mm}$ in zone diameter around disks containing $\beta$ lactamase inhibitors, as compared with the disk with Meropenem alone, is considered to be a positive result for EDTA and Cloxacillin, whereas an increase of $4 \mathrm{~mm}$ for Phenyl Boronic Acid. The associated resistance mechanisms were interpreted based on the table given below.

\section{Results and Discussion}

A total of 430 strains belonging to Enterobacteriacae were obtained from the clinical samples. 60 isolates were found to be Carbapenem resistant (13.95\%) which included $36(60 \%)$ strains of Klebsiella species, 24(40\%) strains of Escherichia coli and one (1.6\%) was Citrobacter freundii. Most of the Carbapenem-resistant isolates were from urine samples.

Among the 4 tests performed KPC Chrom Agar detected Carbapenemases in $58(96.6 \%)$ and MHT in 22(36.6\%) isolates.

Blue Carba Test showed $8(13.3 \%)$ isolates as $\mathrm{KPC} / \mathrm{MBL}$ phenotype and $2(3.3 \%)$ as OXA phenotype.

Inhibitor Based Phenotypic Test detected Carbapenemases in $9(15 \%)$ isolates out of which $5(8.3 \%)$ were MBL producers, 3(5\%) were KPC producers and 1(1.6\%) was Amp $\mathrm{C}$ producer with porin loss.

\begin{tabular}{|l|c|c|c|}
\hline $\begin{array}{l}\text { Carbapenem resistance } \\
\text { mechanism }\end{array}$ & $\begin{array}{c}\text { Meropenem } \\
\text { + Phenyl Boronic Acid }\end{array}$ & $\begin{array}{c}\text { Meropenem } \\
\text { + Cloxacillin }\end{array}$ & $\begin{array}{c}\text { Meropenem } \\
\text { + EDTA }\end{array}$ \\
\hline ESBL or AmpC+porin loss & + & + & - \\
\hline MBL (IMP, NDM, VIM) & - & - & +++ \\
\hline KPC & +++ & - & - \\
\hline
\end{tabular}




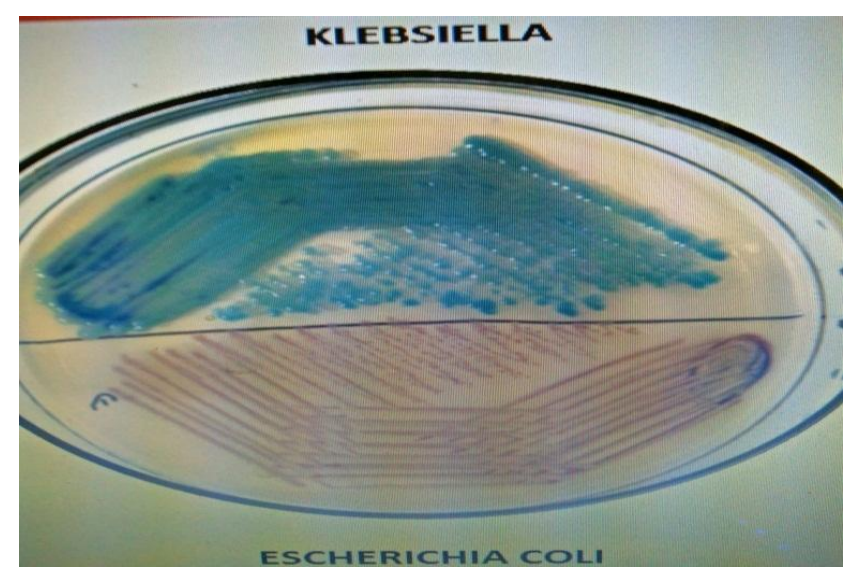

Figure.1 KPC ChromAgar

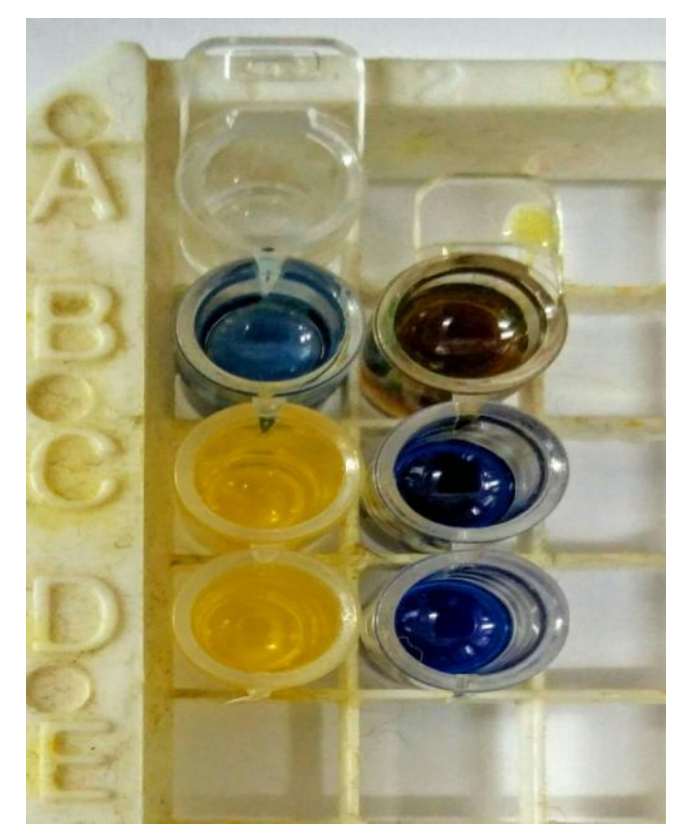

Figure.2 Blue Carba Test

CRE is becoming a significant infection control concern and its appropriate detection has become vital in patient management. Although molecular techniques are regarded as the gold standard for detection of carbapenem resistance, it is difficult to perform in resource constrained laboratories. Hence various phenotypic methods are being used to detect the production of Carbapenemases. In our study we performed screening tests for detection of carbapenemases and supplemental tests to differentiate them into various classes.
Studies have shown KPC ChromAgar to be $100 \%$ sensitive in detecting Carbapenemases and has been recommended for screening CRE. KPC Chrom agar used in our study detected $96.6 \%$ isolates as Carbapenemase producers which correlates with most of the other similar studies.

Though CLSI 2015 recommends Modified Hodge Test (MHT) as one of the confirmatory tests for Carbapenemase detection concerns have been raised over its variable sensitivity and specificity especially 
detecting metallo- $\beta$ - lactamases. Our study detected Carbapenemases production in 22 $(36.6 \%)$ of isolates by MHT.

Carba NP is one of the confirmatory test recommended by CLSI 2016 to detect Carbapenemases. Blue Carba Test, a modified version of Carba NP as proposed by Pires et al., has the advantage of detecting and differentiating Carbapenemases into different classes. Additional advantages compared to Carba NP test were (i) its simplicity due to the direct use of colonies (instead of bacterial extracts); (ii) economical and (iii) useful to detect even OXA-type carbapenemases. According to their study the test, showed $100 \%$ sensitivity and specificity in comparison with the molecular methods. In our study BCT detected Carbapenemases in $20 \%$ of the isolates which showed $13.3 \%$ isolates as $\mathrm{KPC} / \mathrm{MBL}$ phenotype and 3.3\% as OXA phenotype. This test requires further evaluation as the studies using this test are limited.

Inhibitor based phenotypic test is a novel assay that detects different classes of Carbapenemases. The associated resistance mechanisms are interpreted based on the inhibition of Carbapenemases by specific inhibitors. Boronic acid is a potent inhibitor of KPC. EDTA is a potent inhibitor of the zinc-dependent MBL. The AmpC- $\beta$ lactamase is inhibited by both Boronic acid and Cloxacillin. Giske et al., in his study showed that use of PBA and EDTA for phenotypic detection of carbapenemase to be $100 \%$ sensitive, using PCR as gold standard. Dutta et al., reported prevalence of $5.75 \%$ of MBL type of carbapenemase. In our study IBPT detected $8.3 \%$ isolates as KPC producers, 5\% as MBL producers and $1.6 \%$ as AmpC producer.Thus in our study KPC and Metallobetalactamases were the predominant phenotypes.
In conclusion, all the four phenotypic tests were found to be simple and easy to perform. Among these tests KPC ChromAgar could be useful as a screening test for Carbapenemase detection and supplemental tests such as Blue Carba Test and Inhibitor Based Phenotypic Test to differentiate these Carbapenemases. However genotypic tests would be required for confirmation.

\section{References}

CDC. 2014. Healthcare-associated Infections (HAIs) Carbapenemresistant Enterobacteriaceae in Healthcare Settings Available at http://www.cdc.gov/hai/organisms/cr e/ CDC. 2012. CRE Toolkit Guidance for Control of Carbapenem-resistant Enterobacteriacae (CRE).Centers for Disease Control and Prevention, USA. Available at http://www.cdc.gov/hai/organisms/cr e/cre-toolkit/

Clinical and Laboratory Standards Institute. Performance Standards for Antimicrobial Susceptibility Testing: Twenty Sixth Informational Supplement M100-S26. CLSI, Wayne, PA: USA; 2016.

Queenam, A.M., Bush, K. 2007. Carbapenemases: The versatile betalactamases. Clin. Microbiol. Rev., 20: 440-58.

Public Health England. Acute trust toolkit for the early detection, management and control of carbapenemaseproducing Enterobacteriaceae. 2013. Available https://www.gov.uk/government/upl oads/system/uploads/attachment_dat a/file/329227/Acute_trust_toolkit_fo r_the_early_detection.pdf. 
Pravin, K., Nair, Michelle, S., Vaz. Prevalence of carbapenem resistant Enterobacteriaceae from a tertiary care hospital in Mumbai, India. $J$. Microbiol. Infect. Dis., 3(4): 207210.

Jacob Moran Gilada, Yehuda Carmelib, David Schwartza, Shiri NavonVenezia. 2011. Laboratory evaluation of the CHROMagar KPC medium for identification of carbapenem-non-susceptible Enterobacteriaceae.

Diag. Microbiol. Infect. Dis., 70: 565-567.

Modified Hodge Test for Carbapenemase Detection in Enterobacteriacae. Available at http:www.cdc.gov/HAI/pdfs/lab-

Settings/HodgeTest_Carbapenemase _Enterobacteriacae.pdf

Pires, J., Novais, A., Peixe.Blue-Carba, L. 2013. An Easy Biochemical Test for Detection of Diverse Carbapenemase Producers Directly from Bacterial Cultures. J. Clin. Microbiol., 51(12): 4281.
Giske, C.G., Gezelius, L., Samuelsen, M., Warner, A., Sundsfjord, Woodford, N. 2010. A sensitive and specific phenotypic assay for detection of metallo-blactamases and KPC in Klebsiella pneumoniae with the use of meropenem disks supplemented with aminophenylboronic acid, dipicolinic acid and cloxacillin. Microbiology and Infection 2010 European Soc. Clin. Microbiol. Infect. Dis., 17: 552-556.

Datta, P., Gupta, V., Garg, S., Chander, J. 2012. Phenotypic method for differentiation of carbapenemases in Enterobacteriaceae: Study from north India. Indian J. Pathol. Microbiol., 55: 357-360.

Wattal, C., Goel, N., Oberoi, J.K., et al. 2010. Surveillance of multidrug resistant organisms in tertiary care hospital in Delhi, India. J. Assoc. Physicians India, 58: 32-36.

\section{How to cite this article:}

Akshaya Rao and V.A. Indumathi. 2016. Detection of Carbapenem Resistant Enterobacteriacae from Clinical Isolates. Int.J.Curr.Microbiol.App.Sci. 5(5): 864-869. doi: http://dx.doi.org/10.20546/ijcmas.2016.505.089 